\title{
Influencia do uso de chupetas e mamadeiras na prática do aleitamento materno
}

\section{Influence of the use of artificial nipples and baby bottles in breastfeeding}

\author{
Christyann Lima Campos Batista ${ }^{1}$, Valdinar Sousa Ribeiro ${ }^{2}$, Maria do Desterro Soares Brandão Nascimento ${ }^{3}$
}

1. Hospital Universitário da Universidade Federal do Maranhão (HUUFMA), São Luís, MA, Brasil. 2. Docente do Departamento de Medicina III da Universidade Federal do Maranhão (UFMA), São Luís, MA, Brasil. 3. Docente do Programa de Pós-Graduação em Saúde do Adulto e da Criança da Universidade Federal do Maranhão (UFMA), São Luís, MA, Brasil.

\begin{abstract}
Resumo
Objetivo: Verificar, na mais recente literatura, o efeito dos bicos artificiais, como mamadeiras e chupetas, sobre a prática do aleitamento materno. Métodos: Foi realizada uma revisão da literatura nas bases de dados LILACS, SciELO e PubMed, considerando estudos originais publicados entre os anos de 2010 e 2015, em português e inglês, utilizando-se os descritores "chupetas", "amamentação", "mamadeiras", "desmame" e "alimentação artificial", combinados ou isolados, com seus respectivos correspondentes na língua inglesa. Resultados: foram incluídos 25 artigos conforme os critérios de inclusão e exclusão. Estudos apontaram uma relação entre o desmame e o uso de chupetas, e o desmame e uso de chupetas e outros bicos artificiais. Além disso, os estudos evidenciaram que a redução no uso de chupeta gera um aumento na duração do aleitamento. Outras variáveis comuns relacionadas ao desmame relatadas na literatura foram: ausência materna, trabalhar fora ou falta de proteção legal, baixa escolaridade materna, idade materna e problemas relacionados às mamas e nascimento em unidade não "Hospital Amigo da Criança". Conclusões: 0 uso de bicos artificiais está sendo relacionado ao desmame precoce ou à diminuição da duração do aleitamento materno. Necessita-se ainda que outros estudos sejam realizados a fim de compreender melhor como os bicos artificiais agem sobre as habilidades orais da criança.
\end{abstract}

Palavras-chave: Amamentação. Chupetas. Alimentação Artificial. Desmame.

\begin{abstract}
Objective: To verify, in the most recent literature, the effect of artificial nipples, such as baby bottles and pacifiers, on the practice of breastfeeding. Methods: A review of the literature in the LILACS, SciELO and PubMed databases was carried out, considering original studies published between the years 2010 and 2015, in Portuguese and English, using the descriptors "pacifiers", "breastfeeding", "Weaning", and "artificial feeding", combined or isolated. Results: 25 articles were included according to the inclusion and exclusion criteria. Studies have pointed out a relationship between weaning and the use of pacifiers, and the weaning and use of pacifiers and other artificial nipples. In addition, studies have shown that reduction in pacifier use leads to an increase in the duration of breastfeeding. Other common variables related to weaning reported in the literature were maternal absence, working out or lack of legal protection, low maternal schooling, maternal age and problems related to breasts and birth in a non-baby friendly hospital. Conclusions: The use of artificial nipples is being related to early weaning or shortening the duration of breastfeeding. Further studies are needed to better understand how artificial nipples act on a child's oral skills.
\end{abstract}

Key words: Breastfeeding. Pacifiers. Artificial Feeding. Weaning.

\section{INTRODUÇÃO}

Os benefícios a curto e longo prazo da prática de amamentar ratificam a importância da manutenção do aleitamento materno exclusivo para bebês até 6 meses de idade. ${ }^{1}$ Do mesmo modo, a continuidade do aleitamento materno durante a introdução da alimentação complementar, nos primeiros dois anos de idade, consolida uma extensa lista de evidências científicas para o alcance de um crescimento adequado, tendo recomendação consolidada da Organização Mundial da Saúde (OMS). ${ }^{1-3}$

Para tanto, a OMS recomenda a não utilização de bicos artificiais ou chupetas em bebês que amamentam no seio materno, pois esses bicos contribuem para o encerramento precoce da amamentação. Não dar bicos artificiais ou chupetas a crianças amamentadas ao seio tem destaque nos "Dez Passos para o
Sucesso do Aleitamento Materno". 4-6

Ainda assim, a taxas de manutenção do aleitamento materno exclusivo estão longe dessa recomendação. No Brasil a prevalência do AME em menores de 6 meses foi de $41 \%$ no conjunto das capitais brasileiras e Distrito Federal, em pesquisa realizada em 2009.7 Já a duração mediana do AME foi de apenas 54,1 dias, de acordo com os dados da II Pesquisa sobre a prevalência do Aleitamento Materno no Brasil. ${ }^{7}$ Nos Estados Unidos, 19,4\% dos bebês foram amamentados até os 6 meses, porém de forma exclusiva, apenas $18,8 \% .^{8}$ Para o conjunto de 45 estados-membros da OMS na Europa, a proporção de bebês amamentados diminui conforme a idade. A prevalência do AME abaixo dos 6 meses teve média de 23\%. Apenas treze 
países reportaram taxas acima de $20 \%$. As maiores taxas são do Quirguistão (46\%), Georgia (55\%) e Croácia (52\%) e as menores são da Polônia (4\%) e Bulgária (2\%). A amamentação durante 6 meses apresenta taxas ainda piores, tendo média de 13\% para 31 países do bloco que apresentam esses dados. A Eslováquia e a Hungria apresentaram os melhores resultados (49 e $44 \%)$ e a Grécia o pior número, com apenas $0,7 \%$ dos bebês amamentados nos 6 meses de forma exclusiva. ${ }^{9}$ Observa-se que, apesar da reconhecida importância da amamentação, a prática não é comum a nível global seja em países em desenvolvimento ou em países desenvolvidos.

Nesse período, vários fatores podem influenciar o ritmo com o qual os bebês desempenham sucções e pausas, incluindo idade, fome, posição da boca no seio materno, tempo de sucção e pressão, fadiga e saciedade e, ritmo de descida do leite, quando a influência do uso dos bicos artificiais nesses processos refinados de retirada do leite no seio materno requer ser considerada. ${ }^{10}$ Tanto que o uso de chupetas mostra-se associado ao desmame em bebês que nascem com baixo peso, em regiões pobres. Ambiente que mostra a importância da manutenção do Aleitamento Materno no combate as morbimortalidades ${ }^{11}$.

Estudos observacionais têm encontrado associação positiva entre a suplementação e a baixa duração do aleitamento. ${ }^{12}$ As experiências orais precoces, que favoreçam o surgimento de mecanismos de sucção diferentes dos utilizados no aleitamento materno, podem causar alteração na pega e, consequentemente, fracasso no aleitamento. Essas dificuldades são tidas na literatura de amamentação como confusão de bicos. $^{13}$

A confusão de bicos foi definida como a dificuldade do bebê em atingir a configuração oral correta, ou seja, a abertura de boca e posicionamento da língua, a pega adequada e o padrão de sucção necessários para um aleitamento materno eficiente depois de exposição à mamadeira ou a qualquer outro bico artificial. ${ }^{14}$ Entretanto, existe uma preocupação sobre a real influência do uso dos bicos e a cultura de aleitamento. Boa parte desta controvérsia vem da inconsistência nos achados de pesquisa que relacionem a amamentação e o uso de mamadeiras ou chupetas. Disso decorre um longo debate científico se o uso de chupeta e/ou mamadeira pode realmente ser um marcador de dificuldades na amamentação ou um comportamento negativo para a continuidade da amamentação pelas mães. ${ }^{15-17}$

As atuais evidências de alta qualidade não suportam a afirmação de que o uso de chupetas leva a diminuição da duração do aleitamento. Nesse escopo, inclui-se uma revisão Cochrane que objetivou avaliar o efeito do uso de chupeta sobre a duração do aleitamento materno. Concluiu-se que, seja iniciada logo após o nascimento, ou após o estabelecimento da amamentação o uso de chupetas não afetou significativamente a duração ou a prevalência exclusiva ou parcial do aleitamento até os 4 meses de vida dos lactentes, trazendo mais dúvidas e incertezas aos profissionais de saúde. ${ }^{18} \mathrm{O}$ estudo, entretanto, deixa claro que ainda faltam evidências quanto ao possível efeito do uso de chupetas sobre dificuldades maternas imediatas em amamentar, bem como possíveis efeitos a longo prazo do uso de chupetas sobre a saúde dos lactentes.

Sob a ótica deste debate, este artigo tem como tema de pesquisa verificar o efeito dos bicos artificiais, como mamadeiras e chupetas, sobre a prática do aleitamento materno por meio de revisão de literatura. Dessa forma, pais e profissionais de saúde podem ter um ponto de início sobre a decisão de oferecer ou não chupetas e mamadeiras de forma indiscriminada a neonatos.

\section{MÉTODOS}

\section{Fonte de dados}

Trata-se de uma revisão integrativa da literatura. Optou-se por esse método devido à possibilidade de síntese de conhecimento e a incorporação da aplicabilidade de resultados de estudos significativos na prática. ${ }^{19}$

As bases de dados incluídas nas buscas foram: Literatura Latino-Americana e do Caribe em Ciências da Saúde (LILACS); a Scientific Electronic Library Online (SciELO) e a US National Library of Medicine (PubMed).

Para a LILACS e a Scielo, os unitermos utilizados para a seleção das publicações foram "chupetas" AND "amamentação"; "mamadeiras" AND "amamentação"; "desmame" AND "amamentação"; e "amamentação" AND "alimentação artificial”. Para a base de dados PubMed os unitermos foram "pacifiers" AND "breastfeeding"; "nursing bottles" AND "breastfeeding"; "weaning" AND "breastfeeding"; e "breastfeeding" AND "bottle feeding". Só foram considerados os artigos publicados no período compreendido entre 2010 e 2015.

A revisão integrativa compreende cinco etapas: 1) estabelecimento da hipótese ou questão da pesquisa; 2) amostragem; 3) caracterização dos estudos; 4) análise dos resultados; e 5) apresentação e discussão dos achados. ${ }^{20}$ Todas essas etapas foram incluídas no processamento dos estudos encontrados.

Os critérios de seleção dos artigos foram: textos integrais disponíveis em língua portuguesa ou inglesa. Foram excluídos do escopo: artigos que se repetiam entre as bases, artigos unicamente de revisão de literatura e artigos com temáticas não relevantes para responder à questão problema. Para melhor uniformização dos resultados e compreensão das análises, foram excluídos os artigos que envolviam pesquisas com bebês prematuros ou com baixo-peso, lactentes com patologias pré-existentes ou associadas, uso de medicações, anomalias craniofaciais e estudos com gemelares. O objetivo dessa seleção é uniformizar artigos com os efeitos dos bicos artificiais em bebês a termo saudáveis e observar a influência destes na saúde da criança.

Os autores organizaram um instrumento de coleta de dados que auxiliasse na organização do material disponível. A pesquisa 
nas bases de dados aconteceu no mês de fevereiro de 2016. Utilizou-se o aplicativo Mendeley Desktop (versão 1.15.3) para organização catalográfica dos materiais selecionados para análise e também para a geração das referências autorais.

\section{RESULTADOS}

Foram encontrados 6935 artigos, distribuídos conforme Tabela 1. Entretanto, a após a aplicação dos critérios de exclusão, chegouse a um total de 25 artigos que preenchiam completamente o objetivo proposto. A principal meta na análise dos achados foi por ano de publicação, entre 2010 a 2015, relação direta com o tema, ou seja, entre o uso dos bicos artificiais e sua influência sobre o aleitamento materno, além da aplicação dos demais critérios de exclusão como a repetição dos artigos entre as bases de dados. A lista dos artigos está disponível na Tabela 2.

Tabela 1. Relação do número de artigos encontrados, segundo as bases e dados e os unitermos pesquisados.

\begin{tabular}{lrrr}
\hline Unitermos & PubMed & Scielo & LILACs \\
\hline Amamentação e mamadeiras & 173 & 8 & 47 \\
Amamentação e chupetas & 269 & 15 & 84 \\
Amamentação e desmame & 2.258 & 101 & 335 \\
Amamentação e alimentação & 3.491 & 13 & 141 \\
artificial & & & \\
TOTAL & 6.191 & 137 & 607 \\
\hline
\end{tabular}

Tabela 2. Artigos analisados nessa revisão, organizados segundo autor, ano, país; desenho de estudo; localidade, número de participantes, amostragem e período; e objetivo.

\begin{tabular}{|c|c|c|c|}
\hline Autoria, ano de publicação & Desenho & $\begin{array}{l}\text { Localidade, número de participantes } \\
\text { e amostragem e período }\end{array}$ & Objetivo \\
\hline $\begin{array}{l}\text { Caminha M de FC, et al., } \\
2011^{21}\end{array}$ & Transversal & $\begin{array}{l}\text { Recife, Brasil. } 37 \text { mulheres mãe de } \\
\text { crianças }<5 \text { anos. Ano de } 2001 \text {. }\end{array}$ & $\begin{array}{l}\text { Identificar a frequência e o período de aleitamento } \\
\text { materno exclusivo entre profissionais de um } \\
\text { Programa Saúde da Família. }\end{array}$ \\
\hline S. Neto ET, et al., $2013^{22}$ & Coorte populacional & $\begin{array}{l}\text { Vitória, Brasil. } 86 \text { mães e bebês. } \\
\text { Novembro de } 2003 \text { a junho de } 2006\end{array}$ & $\begin{array}{l}\text { Analisar os fatores associados à duração do } \\
\text { aleitamento materno por dois modelos de análise } \\
\text { estatística }\end{array}$ \\
\hline Sousa RV, et al., $2012^{23}$ & Transversal, descritiva & $\begin{array}{l}\text { Riacho Fundo, Brasil. } 174 \text { crianças } \\
\text { menores de seis meses. Maio a Julho } \\
\text { de } 2011 .\end{array}$ & $\begin{array}{l}\text { Verificar atual situação do aleitamento materno } \\
\text { exclusivo e fatores associados à sua interrupção } \\
\text { em menores de } 6 \text { meses }\end{array}$ \\
\hline Rigotti RR, et al., $2015^{24}$ & Transversal & $\begin{array}{l}\text { Barra Mansa, Brasil. Entrevista com } \\
\text { acompanhantes de } 580 \text { crianças de } 6 \\
\text { a } 11 \text { meses. Ano de } 2006 \text {. }\end{array}$ & $\begin{array}{l}\text { Investigar a associação entre uso de mamadeira e } \\
\text { chupeta e o aleitamento materno no } 20 \text { semestre } \\
\text { de vida }\end{array}$ \\
\hline Perrine CG, et al., $2012^{25}$ & Longitudinal & $\begin{array}{l}\text { Estados Unidos. } 1457 \text { Mulheres } \\
\text { recrutadas no 3o trimestre de } \\
\text { gravidez. Entre } 2005 \text { e } 2007 \text {. }\end{array}$ & $\begin{array}{l}\text { Descrever as intenções de aleitamento materno } \\
\text { exclusivo das mães e se as práticas do IHAC estão } \\
\text { associadas com o êxito dessas intenções. }\end{array}$ \\
\hline Carrascoza KC, et al., $2014^{26}$ & Estudo longitudinal, quantitativo. & $\begin{array}{l}\text { Piracicaba, Brasil. Acompanhamento } \\
\text { de } 120 \text { díades. Durante o ano de } 2004 .\end{array}$ & $\begin{array}{l}\text { Avaliar a introdução de chupeta entre crianças } \\
\text { assistidas por um programa interdisciplinar de } \\
\text { incentivo ao aleitamento materno durante os } \\
\text { primeiros seis meses de vida, investigando os } \\
\text { possíveis determinantes do seu uso }\end{array}$ \\
\hline $\begin{array}{l}\text { Rocci E, Fernandes RAQ } \\
2014^{27}\end{array}$ & Estudo de coorte & $\begin{array}{l}\text { São Paulo, Brasil. } \\
225 \text { mães entrevistadas no puerpério } \\
\text { mediato e no } 15 \circ, 30 \circ, 60 \circ, 120 \text { e } \\
180 \text { - dias após a alta. Janeiro a março } \\
\text { de } 2010 \text {. }\end{array}$ & $\begin{array}{l}\text { Verificar o tempo médio de AME de crianças } \\
\text { nascidas em Hospital Amigo da Criança e } \\
\text { correlacioná-lo com as variáveis: estado civil, } \\
\text { idade materna, peso do bebê, dificuldades na } \\
\text { amamentação e orientações recebidas. }\end{array}$ \\
\hline Vieira TO, et al., $2014^{28}$ & Estudo de coorte & $\begin{array}{l}\text { Feira de Santana, Brasil. } 1344 \text { mães e } \\
\text { bebês. Entre abril de } 2004 \text { e maio de } \\
2005 .\end{array}$ & $\begin{array}{l}\text { Identificar os fatores associados com a } \\
\text { descontinuidade do AME. }\end{array}$ \\
\hline Levinienè G, et al., $2013^{29}$ & Longitudinal & $\begin{array}{l}\text { Kaunas, Lithuania. } \\
195 \text { mães. Setembro de } 2008 \text { a } \\
\text { fevereiro de } 2010 .\end{array}$ & $\begin{array}{l}\text { Identificar os fatores associados com a duração do } \\
\text { aleitamento materno. }\end{array}$ \\
\hline $\begin{array}{l}\text { Granville-Garcia AF, et al., } \\
2012^{30}\end{array}$ & Transversal, analítico & $\begin{array}{l}\text { Campina Grande, Brasil. } \\
800 \text { mães de crianças de } 0-24 \text { meses. } \\
\text { Entre outubro de } 2008 \text { a fevereiro de } \\
2009 .\end{array}$ & $\begin{array}{l}\text { Determinar os fatores associados com o desmame } \\
\text { precoce em um Hospital Amigo da Criança. }\end{array}$ \\
\hline Salustiano LPQ, et al., $2012^{31}$ & Transversal & $\begin{array}{l}\text { Uberlândia, Brasil. } 667 \text { crianças de } \\
\text { seis meses que compareceram na } \\
\text { Campanha de Multivacinação. Agosto } \\
\text { de } 2008 \text {. }\end{array}$ & $\begin{array}{l}\text { Descrever as características maternas e das } \\
\text { crianças, bem como avaliar os fatores associados } \\
\text { à interrupção do aleitamento materno exclusivo. }\end{array}$ \\
\hline
\end{tabular}




\begin{tabular}{|c|c|c|c|}
\hline Autoria, ano de publicação & Desenho & $\begin{array}{l}\text { Localidade, número de participantes } \\
\text { e amostragem e período }\end{array}$ & Objetivo \\
\hline Giuliani NR et al., $2011^{32}$ & Transversal & $\begin{array}{l}\text { Florianópolis, Brasil. } 200 \text { mulheres, } \\
\text { mães de bebês de seis a } 12 \text { meses de } \\
\text { idade. Janeiro a abril de } 2005 \text {. }\end{array}$ & $\begin{array}{l}\text { Identificar a prevalência do desmame precoce } \\
\text { (aleitamento materno exclusivo }<6 \text { meses) entre } \\
\text { mães de crianças de seis a } 12 \text { meses de idade. }\end{array}$ \\
\hline $\begin{array}{l}\text { Leone CR, Sadeck L dos SR } \\
2012^{33}\end{array}$ & Transversal, observacional. & $\begin{array}{l}\text { São Paulo, Brasil. } 724 \text { pais/ } \\
\text { responsáveis de crianças } \leq 6 \text { meses. } \\
\text { Agosto de } 2008 \text {. }\end{array}$ & $\begin{array}{l}\text { Avaliar os fatores de risco associados à ausência de } \\
\text { aleitamento materno exclusivo (AME) em crianças } \\
\leq 6 \text { meses de vida. }\end{array}$ \\
\hline Dalili H, et al., $2013^{34}$ & Transversal & $\begin{array}{l}\text { Teerã, Irã } \\
175 \text { mães que compareceram para } \\
\text { uma dose vacinal. } \\
\text { Outubro a dezembro de } 2011 .\end{array}$ & $\begin{array}{l}\text { Avaliar a frequência do AME em dois centros de } \\
\text { saúde. }\end{array}$ \\
\hline Sousa RV, et. al., $2012^{35}$ & Transversal & $\begin{array}{l}\text { Campina Grande, Brasil. } 800 \text { mães } \\
\text { responderam a uma entrevista } \\
\text { estruturada. Julho de } 2008 \text { a } \\
\text { novembro de } 2009\end{array}$ & $\begin{array}{l}\text { Verificar a prevalência e a associação de hábitos de } \\
\text { alimentação e de sucção de bebês, na faixa etária } \\
\text { de zero a } 24 \text { meses. }\end{array}$ \\
\hline Chantry CJ, et al., $2014^{36}$ & Coorte longitudinal & $\begin{array}{l}\text { Sacramento, EUA. } \\
448 \text { primíparas foram avaliadas. Entre } \\
\text { janeiro de } 2006 \text { e dezembro de } 2007 .\end{array}$ & $\begin{array}{l}\text { Avaliar o uso de formulas no hospital entre } \\
\text { mães primíparas que pretendiam amamentar } \\
\text { exclusivamente e verificar determinar se esse uso } \\
\text { encurta a duração da amamentação. }\end{array}$ \\
\hline Kair LR, et al., $2012^{37}$ & Retrospectivo & $\begin{array}{l}\text { Portland, EUA. } 2249 \text { bebês que foram } \\
\text { admitidos na unidade mãe-bebê. } \\
\text { Entre julho de } 2010 \text { e agosto de } 2011\end{array}$ & $\begin{array}{l}\text { Testar a hipótese de que ao remover a distribuição } \\
\text { de chupetas na unidade mãe-bebê está associada } \\
\text { ao um aumento no AME ou melhor início do AM }\end{array}$ \\
\hline Mauch CE, et al., $2012^{38}$ & Longitudinal & $\begin{array}{l}\text { Adelaide, Australia. } 670 \text { primíparas } \\
\text { responderam a um questionário } \\
\text { sobre uso de chupetas e alimentação } \\
\text { dos bebês. Fevereiro a junho de 2008; } \\
\text { setembro a março de } 2009 \text {. }\end{array}$ & $\begin{array}{l}\text { Investigar quem (se alguém) advertiu as primíparas } \\
\text { sobre o uso de chupetas e as razões porque elas } \\
\text { ofereceram (ou tentaram dar) a chupeta aos seus } \\
\text { bebês a termo. }\end{array}$ \\
\hline Queluz MC et al., $2012^{39}$ & Transversal e quantitativo & $\begin{array}{l}\text { Serrana, Brasil. } 275 \text { crianças menores } \\
\text { de } 6 \text { meses. Setembro de } 2009 \text {. }\end{array}$ & $\begin{array}{l}\text { Identificar a prevalência e os determinantes } \\
\text { do aleitamento materno exclusivo em crianças } \\
\text { menores de } 6 \text { meses }\end{array}$ \\
\hline $\begin{array}{l}\text { Orimadegun } \mathrm{AE} \text {, Obokon } \mathrm{GO} \\
2015^{40}\end{array}$ & Transversal & $\begin{array}{l}\text { Ibadan North Local Government Area } \\
\text { (IBNLGA), Nigeria. Entrevista com } 427 \\
\text { mães de crianças com idade entre } 6 \text { e } \\
23 \text { meses. Ano de } 2006 \text {. }\end{array}$ & $\begin{array}{l}\text { Determinar a prevalência de hábitos de hábitos } \\
\text { de sucção não-nutritiva e associação com diarreia } \\
\text { aguda. }\end{array}$ \\
\hline Castillo SD, et al., $2012^{41}$ & Transversal & $\begin{array}{l}\text { Campinas, Brasil. } 642 \text { crianças de } 0-12 \\
\text { meses atendidas em um hospital. } \\
\text { Agosto de } 2009 \text { a março de } 2010 .\end{array}$ & $\begin{array}{l}\text { Avaliar a prevalência do uso da chupeta em relação } \\
\text { ao tipo de aleitamento, as razões das mães para } \\
\text { introduzi-la ou não e os fatores associados à } \\
\text { mudança de opinião quanto à sua intenção inicial } \\
\text { a esse respeito. }\end{array}$ \\
\hline Vieira GO, et al., $2015^{42}$ & Transversal & $\begin{array}{l}\text { Feira de Santana, Brasil } \\
\text { Análise de três inquéritos que } \\
\text { envolveram } 5949 \text { menores de um } \\
\text { ano.1996, } 2001 \text { e } 2009 .\end{array}$ & $\begin{array}{l}\text { Verificar a tendência dos indicadores de AM e } \\
\text { identificar características da população que } \\
\text { possam ter influenciado essa tendência. }\end{array}$ \\
\hline Demitto MO, et al., $2013^{43}$ & Transversal & $\begin{array}{l}\text { Maringá, Brasil. } 362 \text { mães. } \\
\text { Entre outubro de } 2010 \text { a março de } \\
2011\end{array}$ & $\begin{array}{l}\text { Comparar o tempo de Aleitamento Materno } \\
\text { Exclusivo (AME) entre os grupos de idade de } \\
\text { início do uso da chupeta e verificar a presença de } \\
\text { associação entre o uso da chupeta e a interrupção } \\
\text { precoce do AME. }\end{array}$ \\
\hline Buckstegge AK, et al., $2014^{44}$ & Transversal, retrospectivo & $\begin{array}{l}\text { Almirante Tamandaré, Brasil. } 124 \\
\text { mães de crianças com até } 12 \text { meses } \\
\text { de idade. Junho a setembro de } 2011\end{array}$ & $\begin{array}{l}\text { Analisar os fatores associados ao desmame em } \\
\text { crianças sul brasileiras de comunidades de baixa } \\
\text { renda }\end{array}$ \\
\hline $\begin{array}{l}\text { Martins EJ, Giuliani ERJ } \\
2012^{45}\end{array}$ & Estudo de coorte & $\begin{array}{l}\text { Porto Alegre, Brasil. } 151 \text { crianças do } \\
\text { nascimento até a idade de } 3 \text { a } 5 \text { anos. } \\
\text { Entre os meses de junho e novembro } \\
\text { de } 2003\end{array}$ & $\begin{array}{l}\text { Identificar fatores associados à manutenção do } \\
\text { aleitamento materno por } 2 \text { anos ou mais. }\end{array}$ \\
\hline
\end{tabular}

A maior parte dos estudos incluídos nessa revisão associam diretamente o desmame precoce (ou a descontinuidade do aleitamento materno) ao uso da chupeta. $21,22,24,25,26,28,30,31,33,35,39,42,43,44,45$ Ressaltamos que, entre esses estudos, os autores analisam tanto em períodos de aleitamento materno exclusivo, quando após os 180 dias, preconizados pela OMS. O segundo maior achado foi o que associou o uso de mamadeiras ou a prática da alimentação artificial ao desmame. ${ }^{22,24,25,30,34,35,36,45}$

A prática do uso de chupetas e de uso de alimentação artificial foi também relatada como fator influenciador da prática do 
aleitamento materno, mas, nesse caso, está associado a uma menor duração do aleitamento materno..$^{22,29,38} \mathrm{Um}$ estudo descritivo não demonstrou influência dos bicos apesar de apontar um percentual de uso dentro de sua população. ${ }^{23}$

Outro estudo apontou que a utilização de bico artificial não se manteve associada ao desmame precoce no modelo de regressão logística múltipla. ${ }^{32}$

Outros fatores que influenciam o desmame encontrados na literatura estão apontadas no quadro 1.

Quadro 1. Variáveis mais prevalentes na associação com o desmame ou com a menor duração do aleitamento materno.

1. Mãe não estar presente durante o período de aleitamento
materno, trabalha fora ou trabalha sem proteção legal para
AM $^{24,28,31,32,39,45}$
2. Idade materna ${ }^{32,39,40,42}$
3. Baixa escolaridade materna ${ }^{24,29,28,42}$
4. Problemas mamários ${ }^{28,29,34}$
5. Nascimento em unidade não credenciada Hospital Amigo da
Criança ${ }^{27,28}$
6. Baixo peso ao nascer ${ }^{24,30}$

Já com relação aos tipos de estudo, o mais prevalente foi o transversal utilizado em $60 \%$ dos estudos $(15)^{21,23,24,30-35,39-}$ 44 , seguidos por coortes longitudinais $(9)^{22,25-29,36,38,45}$ e um retrospectivo ${ }^{37}$. O número de estudos transversais aponta para a dificuldade na associação entre etiologia e a manifestação da doença, limitação inerente a este desenho.

Em síntese, observa-se que o uso dos bicos artificiais está relacionado a alterações no processo natural de estabelecimento do aleitamento materno, trazendo consigo dificuldades relacionadas diretamente ao desmame ou a outros fatores influenciadores da não continuidade do ato de amamentar. Este achado demonstra que o uso desses bicos durante o período de aleitamento materno exclusivo representa em si um risco para o não alcance da duração ideal do tempo de amamentação, trazendo consigo prejuízos para a saúde da criança, da mãe e da coletividade que os cerca.

A pesquisa alerta para queixas trazidas por mães para o profissional especializado comuns no aleitamento como baixo peso ou baixo ganho ponderal, queixas mamárias, infecções por fungos ou bactérias no bebê, desnutrição ou desidratação, as quais são diretamente correlacionadas com a precária ingesta de leite humano podem ter como fator coadjuvante o uso de mamadeiras ou chupetas. Entretanto, a principal limitação dos artigos avaliados é a prevalência de estudos transversais, que não permitem situar uma relação temporal entre a exposição e o efeito, dificultando o estabelecimento de uma relação causal. Ainda por cima, não se verificaram, neste estudo, os efeitos a longo prazo que são prevalentes na clínica da saúde da criança como hábitos deletérios, problemas oclusais e sua relação com aspectos respiratórios e de aprendizagem na primeira infância. Outro achado foi a mãe não estar presente durante o período de aleitamento materno, trabalhar fora ou, ainda, trabalhar sem proteção legal para AM, associado ao desmame. Questionase, portanto, a necessidade de melhor cobertura das leis trabalhistas nacionais e internacionais como fator de proteção para a amamentação ou a duração ótima do aleitamento materno.

Da mesma maneira, as dificuldades precoces que se manifestam no período em que a lactação ainda está sendo estabelecida constituem fatores impeditivos para o AME. Esses problemas envolvem distúrbios decorrentes do acúmulo de leite das mamas por má sucção do bebê, levando a quadros como o ingurgitamento mamário podendo progredir para outros problemas como fissuras mamilares e mastite. Indaga-se até que ponto o uso dos bicos possa influenciar na predisposição para os problemas mamários, sendo que ainda se encontra em investigação o real impacto do uso dos bicos e as características de sucção do recém-nascido. O fator idade materna é um que não se encontra consolidado, pois os estudos não são conclusivos na relação da idade e a duração do aleitamento materno. Suscita-se a necessidade de estudos com metodologia mais apropriada que estabeleçam essa relação causal num estudo de seguimento.

\section{Uso dos bicos: é possível alcançar um equilíbrio?}

Para a OMS e o Ministério da Saúde, a recomendação de manter o aleitamento materno exclusivo por seis meses e complementado até os dois anos ou mais é uma estratégia primordial. Garantir a saúde da criança, reduzir a morbimortalidade principalmente em países em desenvolvimento são ações que afetam a sociedade e a economia. Entretanto, muitos países, incluindo o Brasil, não conseguem aproximar-se dessa meta.

Os motivos que causam o desmame são diversos, muitos deles já levantados nos resultados desta revisão. O principal, que foi o objeto dessa análise, é o uso dos bicos artificiais. 0 uso de artifícios para saciar a necessidade de sucção do bebê tem um contexto histórico amplo e uma cultura enraizada nas famílias. O hábito de recorrer à suplementação via mamadeira ou chupeta como método de auto-consolo se tornou mais intenso com as transformações econômicas ocorridas a partir da Revolução Industrial do século XVIII, principalmente com a maior inserção da mulher no mercado de trabalho e com a descoberta do processo de pasteurização do leite, esterilização de objetos, noções de higiene e refrigeração, no início do século $\mathrm{XX} .^{46,47}$

Os conhecimentos atuais relacionados aos benefícios da amamentação levaram à implementação de políticas que regulam a propaganda dos substitutos do leite com o objetivo de aumentar as taxas de prevalência de aleitamento garantindo que as crianças tenham uma maior possibilidade de crescimento e desenvolvimento. ${ }^{48}$ Esse movimento cresceu a partir das décadas de 1960 e 1970 no Brasil e nos Estados Unidos, 
quando teve início um movimento em favor da retomada da amamentação. ${ }^{47}$

Pesquisadores e estudiosos em amamentação e em saúde da criança tentam entender os mecanismos que alteram a sucção dos bebês atingidos pelos bicos artificiais, a associação com o desmame e as consequências geradas ao longo da vida, como cáries, má-oclusões, hábitos deletérios e crescimento desarmônico dentário ou facial. E, em grande monta, estão conseguindo realizar essas associações. Boa parte dos achados entre estudos encontrados que não foram incluídos utilizando-se os descritores desta revisão revelou uma grande quantidade de artigos que associam bicos com problemas de desenvolvimento orofacial.

Esta revisão se soma ao escopo de estudos que confirmam a influência do uso de chupeta ou de mamadeira, seja nas instituições de saúde ou no ambiente familiar, gerando dificuldades para o estabelecimento ou continuidade da prática de aleitamento materno, dado que o uso dos bicos artificiais pode levar a uma diminuição na frequência de sucção que cria um efeito fisiológico negativo na estimulação mamária e, desse modo, diminuir a produção de leite. ${ }^{49}$

Os prejuízos podem ser em curto e em longo prazo, tendo em vista que esses hábitos se mantêm elevados entre as famílias. A prevalência do uso de mamadeira e de chupeta no Brasil foi de 58,4 e $42,6 \%$, respectivamente. ${ }^{7}$ A prática materna, também deve ser levada em conta para esse alto índice. A insegurança levada pela crença de "não produzir leite suficiente", "leite fraco" aponta diretamente como possível causa para a complementação precoce, e destarte, justificam a introdução precoce de outros alimentos, a oferta de chupeta e mamadeira, ou mesmo a interrupção do aleitamento materno. ${ }^{50,7}$

Apesar de não encorajar o uso de bicos, algumas recomendações oficiais não vetam totalmente o uso de chupeta, apontando benefícios. É o caso da Canadian Paediatric Society, que sugere que os centros de saúde reconheçam o uso de chupeta como uma opção dos pais, determinadas pelas necessidades da criança ${ }^{51}$, posicionamento semelhante ao da Sociedade Brasileira de Pediatria, que recomenda que os pais tenham claramente esta visão de "prós e contras" do uso da chupeta, para que, junto ao pediatra, possam tomar uma decisão informada quanto a oferecê-la, ou não, a seus bebês. ${ }^{52}$

Muitos pais buscam as informações fornecidas em sites de sociedades científicas as respostas para questionamentos como este: a decisão de dar ou não chupeta para acalentar seus filhos, fato que nem sempre é respondido de forma satisfatória, por ainda existirem dúvidas sobre os reais impactos do uso desse tipo de bico sobre o aleitamento imediato.
Por outro lado, a American Academy of Pediatrics Section on Breastfeeding recomenda evitar o uso de chupeta em bebês amamentados até que a amamentação esteja completamente estabelecida, aproximadamente em três ou quatro semanas após o nascimento. ${ }^{53}$

O contraste estabelecido entre essas recomendações de entidades médicas e a preconização da Organização Mundial da Saúde mostram como estamos distantes de conseguir uma definição formal para o problema. O que se tem de rotina, de forma geral, principalmente em instituições que são acreditadas como "amigas da criança" é o completo seguimento dos "Dez Passos para o Sucesso do Aleitamento Materno"5-6 que recomenta evitar o uso de leite suplementar e não oferecimento de bicos artificiais para bebês amamentados.

As recomendações que não desencorajam o uso de chupetas são em muito influenciadas pelo resultado da meta-análise Cochrane que mostrou que em bebês saudáveis e a termo, o uso de chupeta, em específico não diminui a duração do aleitamento. Porém, a mesma revisão não é conclusiva sobre os potenciais danos em bebês $>4$ meses de idade. $O$ uso prolongado dos bicos aumenta o risco de otite média aguda, candidíase oral e má-oclusão dentária. Além disso, os demais estudos, apesar de na maioria transversais, elencados nesta revisão deixam clara a associação do desmame com o uso dos bicos artificiais. Apesar de a revisão ser considerada um bom nível de evidência científica, as conclusões advieram de apenas dois ensaios clínicos randomizados, e com critérios de inclusão e exclusão muito restritos reduzindo muito a seleção de participantes, fato que pode limitar a validade externa das conclusões.

Nota-se que existem evidências sobre a influência destes bicos no aleitamento materno. Embora não haja dúvidas de que o desmame precoce ocorra com mais frequência entre as crianças que usam chupeta, ainda não são totalmente conhecidos os mecanismos envolvidos nessa associação, necessitando que a comunidade científica se debruce ainda mais sobre este tema a fim de dar luz aos questionamentos que ainda são persistentes.

\section{CONCLUSÃO}

Esta revisão da literatura mostrou que, na maioria dos estudos transversais realizados, a variável uso de chupeta isolado ou em conjunto com outras variáveis e o uso de mamadeira associado ao uso de chupeta e com outras variáveis constituem fator de risco para o desmame precoce e/ou para a diminuição do tempo de duração de aleitamento materno. Entretanto, é necessário que a comunidade científica continue se debruçando sobre o tema, principalmente sobre as variáveis tempo de uso, frequência de uso e quantidade de suplemento suficientes para o não sucesso da amamentação.

\section{REFERÊNCIAS}

1. Horta BL, Victora CG. Short-term effects of breastfeeding: a systematic review on the benefits of breastfeeding on diarrhea and pneumonia mortality.
Genebra: WHO; 2013 [cited 2016 dec 2]. 54p. Available from: http://www. who.int/maternal_child_adolescent/documents/breastfeeding_short_term_ 
effects/en/

2. Horta BL, Victora CG. Long-term health effects of breastfeeding: A SYSTEMATIC REVIEW. World Heal Organ [Internet]. 2013; Available from: (http://www.who. int/maternal_child_adolescent/documents/breastfeeding_long_term_effects/ en/)

3. Mclnnes R, Hoddinott P, Britten J, Darwent K, Craig L. Significant others, situations and infant feeding behaviour change processes: a serial qualitative interview study. BMC Pregnancy Childbirth[Internet]. 2013 [cited 2016 nov 21]; 13(1): 114. Available from: https://bmcpregnancychildbirth.biomedcentral com/articles/10.1186/1471-2393-13-114.

4. Kramer MS, Kakuma R. Optimal duration of exclusive breastfeeding. Cochrane database Syst Rev[Internet]. 2002 [cited 2016 feb 3]; (1): CD003517. Available from: http://www.ncbi.nlm.nih.gov/pubmed/11869667.

5. World Health Organization. Evidence for the Ten Steps to Successful Breastfeeding[Internet]. Geneva: WHO; 1998 [cited 2016 feb 3]. 118p. Available from: http://www.babyfriendlyusa.org/eng/10steps.html.

6. USA BF. The Ten Steps to Successful Breastfeeding [Internet]. Baby Friendly Hospital Initiative. 2012 [cited 2016 feb 2]. Available from: http://www. babyfriendlyusa.org/about-us/baby-friendly-hospital-initiative/the-ten-steps.

7. Ministério da Saúde (BR), Secretaria de Atenção à Saúde, Departamento de Ações Programáticas Estratégicas. II Pesquisa de Prevalência de Aleitamento Materno nas Capitais Brasileiras e Distrito Federal. Brasília: Ministério da Saúde; 2009. 108 p.

8. Centers for Disease Control and Prevention. Breastfeeding Report Card United States 2014. Atlanta: CDC; 2014 [cited 2016 feb 3]. p. 1-8. Available from: http://www.cdc.gov/breastfeeding/pdf/2014breastfeedingreportcard. pdf.

9. Bagci Bosi AT, Eriksen KG, Sobko T, Wijnhoven TM, Breda J. Breastfeeding practices and policies in WHO European Region Member States. Public Health Nutr [Internet]. 2015 [cited 2016 mar 1];19(16): 1. Available from: http://www. journals.cambridge.org/abstract_S1368980015001767.

10. Moral A, Bolibar I, Seguranyes G, Ustrell JM, Sebastiá G, Martínez-Barba $C$, et al. Mechanics of sucking: comparison between bottle feeding and breastfeeding. BMC Pediatr [Internet]. 2010;10:6. Available from: http://www. ncbi.nlm.nih.gov/pubmed/20149217\%5Cnhttp://www.pubmedcentral.nih. gov/articlerender.fcgi?artid=PMC2837866.

11. Cunha AJ, Leite AM, Machado MM. Breastfeeding and pacifier use in Brazil. Indian J Pediatr[Internet]. 2005 [cited 2016 mar 1]; 72(3): 209-12. Available from: http://www.ncbi.nlm.nih.gov/pubmed/15812114.

12. Smith HA, Becker GE. Early additional food and fluids for healthy breastfed full-term infants. Smith HA, editor. Cochrane Database Syst Rev[Internet] 2016 Aug 30 [cited 2016 mar 1]; 2016(8). Available from: http://doi.wiley. com/10.1002/14651858.CD006462.pub4.

13. Medeiros AMC, Bernardi AT. Alimentação do recém-nascido pré-termo: aleitamento materno, copo e mamadeira. Rev da Soc Bras Fonoaudiol[Internet] 2011 [cited 2016 mar 1]; 16(1):73-9. Available from: http://www.scielo.br/ scielo.php?script=sci_arttext\&pid=S1516-80342011000100014\&lng=pt\&nrm=i so\&tlng=pt.

14. Neifert M, Lawrence R Seacat J. Nipple confusion: toward a formal definition. J Pediatr. 1995 Jun; 126(6): S125-9.

15. Victora CG, Tomasi E, Olinto MTA, Barros FC. Use of pacifiers and breastfeeding duration. Lancet. 1993 Feb; 341(8842):404-6. PubMed PMID: 8094171.

16. Ford RP, Mitchell E, Scragg RK, Stewart AW, Taylor BJ, Allen EM. Factors adversely associated with breast feeding in New Zealand. J Paediatr Child Health[Internet]. 1994 Dec [cited 2016 may 23]; 30(6):483-9. Available from: http://www.ncbi.nlm.nih.gov/pubmed/7865258.
17. Jenik AG, Vain NE, Gorestein AN, Jacobi NE, Pacifier and Breastfeeding Trial Group. Does the Recommendation to Use a Pacifier Influence the Prevalence of Breastfeeding? J Pediatr. 2009 Sep;155(3): 350-4. doi: 10.1016/j. jpeds.2009.03.038.

18. Jaafar SH, Ho JJ, Jahanfar S, Angolkar M. Effect of restricted pacifier use in breastfeeding term infants for increasing duration of breastfeeding. Jaafar $\mathrm{SH}$, editor. Cochrane Database Syst Rev [Internet]. Chichester, UK: John Wiley \& Sons, Ltd; 2016 Aug 30;(8). Available from: http://doi.wiley.com/10.1002/14651858. CD007202.pub4 doi: 10.1002/14651858.CD007202.pub4.

19. Souza MT, Silva MD, Carvalho R. Revisão integrativa: o que é e como fazer. Einstein. 2010; 8(1):102-6.

20. Ganong LH. Integrative reviews of nursing research. Res Nurs Health[Internet]. 1987 Feb [acesso 2016 feb 5]; 10(1):1-11. Available from: http://www.ncbi.nlm. nih.gov/pubmed/3644366. PubMed PMID: 3644366.

21. Caminha MFC, Serva VB, Anjos MMR, Brito RBS, Lins MM, Batista M Filho. Aleitamento materno exclusivo entre profissionais de um Programa Saúde da Família. Cien Saude Colet[Internet]. 2011 Abr [acesso 2016 feb 10]; 16(4):2245-50. Available from: http://www.scielo.br/scielo.php?script=sci_ arttext\&pid=S1413-81232011000400023\&Ing=en\&nrm=iso\&tlng=pt. doi: http://dx.doi.org/10.1590/S1413-81232011000400023.

22. S Neto ET, Zandonade E, Emmerich AO. Analysis models for variables associated with breastfeeding duration. Rev Paul Pediatr[Internet]. 2013 Sep; 31(3):306-14. Available from: http://www.ncbi.nlm.nih.gov/ pubmed/24142312\%5Cnhttp://www.scielo.br/scielo.php?script=sci_ arttext\&pid=S0103-05822013000300306\&lng=en\&nrm=iso\&tlng=en. doi: 10.1590/S0103-05822013000300006.

23. Souza NKT, Medeiros MP, Silva MA, Cavalcanti SB, Dias RDS, Valente FA. Aspectos envolvidos na interrupção do aleitamento materno exclusivo. Comum. ciênc. saúde. 2011; 22(4):231-8.

24. Rigotti RR, Oliveira MI, Boccolini CS. Association between the use of a baby's bottle and pacifier and the absence of breastfeeding in the second six months of life. Cien Saude Colet[Internet]. 2015 Abr; 20(4): 1235-44. Available from: http://www.ncbi.nlm.nih.gov/pubmed/25923634. doi: 10.1590/141381232015204.00782014.

25. Perrine CG, Sacanlon KS, Li R, Odom E, Grummer-Strawn LM. Baby-Friendly hospital practices and meeting exclusive breastfeeding intention. Pediatrics. 2012 May; 130(1):54-60.

26. Carrascoza KC, Possobon RDF, Ambrosano GMB, Costa AL Junior, Moraes $A B A$. Determinants of pacifier use among infants attending an interdisciplinary breastfeeding promotion program. Rev CEFAC. 2014 Mar-Abr; 16(2):582-91. doi: http://dx.doi.org/10.1590/1982-021620149712.

27. Rocci E, Fernandes RAQ. Dificuldades no aleitamento materno e influência no desmame precoce. Rev Bras Enferm [Internet]. 2014 Jan-Fev [cited 2016 feb 15]; 67(1): 22-7. Available from: http://www.gnresearch.org/doi/10.5935/00347167.20140002. doi: http://dx.doi.org/10.5935/0034-7167.20140002.

28. Vieira TO, Vieira GO, Oliveira NF, Mendes CM, Giugliani ER, Silva LR Duration of exclusive breastfeeding in a Brazilian population: new determinants in a cohort study. BMC Pregnancy Childbirth [Internet]. 2014 [cited 2016 mar 1]; 14: 175. Available from: http://www.pubmedcentral.nih.gov/ articlerender.fcgi?artid $=4046501 \&$ tool $=$ pmcentrez $\&$ rendertype=abstract. doi: 10.1186/1471-2393-14-175.

29. Levinienè G, Tamulevičienè E, Kudzytè J, Petrauskienè A, Zaborskis A Aželienè I, et al. Factors associated with breastfeeding duration. Medicina (Kaunas)[Internet]. 2013 [cited 2016 mar 15]; 49(9):415-21. Available from: http://www.ncbi.nlm.nih.gov/pubmed/24589578.

30. Granville-Garcia A, Lins RDAU, Oliveira ACB, Paiva SM, Sousa R V, Martins $\checkmark$, et al. Factors associated with early weaning at a Child-Friendly Healthcare Initiative Hospital. Rev. odonto ciênc,[Internet]. 2012 [cited 2016 fev 20]; 
27(3): 202-7. Available from: http://www.scielo.br/scielo.php?script=sci_ arttext\&pid=S1980-65232012000300005.

31. Salustiano LPQ, Diniz ALD, Abdallah VOS, Pinto RDMC. Fatores associados à duração do aleitamento materno em crianças menores de seis meses. Rev Bras Ginecol e Obs. 2012; 34(1):28-33. doi: http://dx.doi.org/10.1590/S010072032012000100006 .

32. Giuliani N de R, de Oliveira J, Traebert J, Santos BZ, Bosco VL. Fatores associados ao desmame precoce em mães assistidas por serviços de puericultura de Florianópolis/SC. Pesqui Bras Odontopeda Clin Integr. 2011 JulSet; 11(3):417-23. doi: 10.4034/PBOCI.2011.113.17.

33. Leone CR, Sadeck LSR, Programa Rede de Proteção a mãe paulistana. Fatores de risco associados ao desmame em crianças até seis meses de idade no município de São Paulo. Rev Paul Pediatr. 2012; 30(1):21-6. doi: http://dx.doi. org/10.1590/S0103-05822012000100004.

34. Dalili H, Farsar A, Barakati H, Raji F, Shariat M, Pourmalek F, et al. Frequency of Exclusive Breastfeeding and Its Affecting Factors in Tehran, 2011. Acta Med Iran. 2014; 52(7):552-6. PubMed PMID: 25135265.

35. Sousa RV, Ferreira JMS, Silva MSP da, Menezes VA, Fontes LBC, GranvilleGarcia AF, et al. Hábitos de alimentação e sucção de bebês assistidos em Hospital Amigo Da Criança, Campina Grande/PB Brasil. Pesqui. bras. odontopediatria clin. integr[Internet]. 2012 Jul [cited 2016 may 1]; 12(2): 10-5. Available from: http://revista.uepb.edu.br/index.php/pboci/article/viewFile/1143/846.

36. Chantry CJ, Dewey KG, Peerson JM, Wagner EA, Nommsen-Rivers LA. InHospital Formula Use Increases Early Breastfeeding Cessation Among First-Time Mothers Intending to Exclusively Breastfeed. J Pediatr; 164(6): 1339-1345. doi: http://dx.doi.org/10.1016/j.jpeds.2013.12.035.

37. Kair LR, Kenron D, Etheredge K, Jaffe AC, Phillipi CA. Pacifier restriction and exclusive breastfeeding. Pediatrics[Internet]. 2013 [cited 2016 mar 30]; 131(4):e1101-7. Available from: http://www.ncbi.nlm.nih.gov/ pubmed/23509161.

38. Mauch CE, Scott JA, Magarey AM, Daniels LA. Predictors of and reasons for pacifier use in first-time mothers: an observational study. BMC Pediatr[Internet]. BMC Pediatrics; 2012 [cited 2016 mar 25];12(1):7. Available from: http://www. biomedcentral.com/1471-2431/12/7. doi: 10.1186/1471-2431-12-7.

39. Queluz MC, Pereira MJB, dos Santos CB, Leite AM, Ricco RG. Prevalence and determinants of exclusive breastfeeding in the city of Serrana, São Paulo, Brazil. Rev. esc. enferm. 2012 Jun; 46(3):537-43. doi: http://dx.doi.org/10.1590/ S0080-62342012000300002.

40. Orimadegun AE, Obokon GO. Prevalence of Non-Nutritive Sucking Habits and Potential Influencing Factors among Children in Urban Communities in Nigeria. Front Pediatr[Internet]. 2015 Apr [cited 2016 mar 25]; 3: 30. Available from: http://www.pubmedcentral.nih.gov/articlerender. fcgi ?artid=4403298\&tool=pmcentrez\&rendertype=abstract. doi: 10.3389/ fped.2015.00030

41. Castilho SD, Casagrande RC, Rached CR, Nucci LB. Prevalência do uso de chupeta em lactentes amamentados e não amamentados atendidos em um hospital universitário. Rev Paul Pediatr. 2012 Jun; 30(2):166-72. doi: http:// dx.doi.org/10.1590/S0103-05822012000200003.
42. Vieira GO, Reis MR, Vieira TO, Oliveira NF, Silva LR, Giugliani ERJ. Trends in breastfeeding indicators in a city of northeastern Brazil. J Pediatr (Rio J)[Internet]. 2015 Maio-Jun [cited 2016 mar 21]; 91(3): 270-7. Available from: http://www. scielo.br/scielo.php?script=sci_arttext\&pid=S0021-75572015000300270. doi: http://dx.doi.org/10.1016/j.jpedp.2015.03.004.

43. Demitto MO, Bercini LO, Rossi RM. Uso de chupeta e aleitamento materno exclusivo. Esc. Anna Nery. 2013 Abr-Jun;17(2):271-6. doi: http://dx.doi. org/10.1590/S1414-81452013000200010.

44. Buckstegge AK, Assunção LR da S, Ferreira FM, Fraiz FC, Boguszewski MCS. Weaning and associated factors in children from low-income communities. Rev odontol. UNESP[Internet]. 2014 May-Jun; 43(3): 172-9. Available from: http://www.scielo.br/scielo.php?script=sci_arttext\&pid=S180725772014000300172\&lang=pt.

45. Martins EJ, Giugliani ER. Which women breastfeed for 2 years or more? J Pediatr (Rio J). 2012 Jan-Feb; 88(1):67-73.

46. Stevens EE, Patrick TE, Pickler R. A history of infant feeding. J Perinat Educ[Internet]. 2009 [cited 2016 mar 23]; 18(2):32-9. Available from: http://www.ncbi.nlm.nih.gov/pubmed/20190854\%5Cnhttp://www. ncbi.nlm.nih.gov/pmc/articles/PMC2684040/pdf/jpe-18-032.pdf. doi: $10.1624 / 105812409 \times 426314$.

47. Castilho SD, Barros ADA Filho, Cocetti M. Evolução histórica dos utensílios empregados para alimentar lactentes não amamentados. Cien saude coletiva. 2010; 15(suppl. 1):1401-10. doi: http://dx.doi.org/10.1590/S141381232010000700050 .

48. Castilho SD, Barros AA Filho. The history of infant nutrition. J Pediatr (Rio J)[Internet]. 2010 May-Jun [cited 2016 mar 30]; 86(3):179-88. Available from: http://www.scielo.br/pdf/jped/v86n3/a04v86n3.pdf.

49. Ministério da Saúde [BR]. Saúde da criança: nutrição Infantil: aleitamento materno e alimentação complementar [Internet] Brasília: Ministério da Saúde; 2009 [cited 2016 mar 30]. 112 p. Available from: http://bvsms.saude.gov. br/bvs/publicacoes/saude_crianca_nutricao_aleitamento_alimentacao.pdf. Caderno de Atenção Básica, 23.

50. Marques ES, Cotta RMM, Priore SE. Mitos e crenças sobre o aleitamento materno. Cienc saúde coletiva. 2011 May; 16(5):2461-8. doi: http://dx.doi. org/10.1590/S1413-81232011000500015

51. Ponti M, Baxter C, James W, Leduc D, Mutch C, Spigelblatt L, et al. Recommendationsfortheuseofpacifiers. PaediatrChild Health[Internet].20030ct [cited 2016 mar 5]; 8(8):515-28. Available from: http://www.pubmedcentral.nih. gov/articlerender.fcgi?artid=2791559\&tool=pmcentrez\&rendertype=abstract

52 Departamento de Pediatria Ambulatorial e Departamento de Aleitamento Materno da SBP. [homepage on the Internet] Uso de chupeta. Conversando com o Pediatra. Cited [2016 oct 14]. Available from: http://www. conversandocomopediatra.com.br/website/paginas/materias_gerais/ materias_gerais.php?id=77\&content=detalhe.

53. American Academy of Pediatrics. Breastfeeding and the Use of Human Milk. Pediatrics. 2012 Mar; 129(3): e827-841.

\section{Como citar este artigo/How to cite this article:}

Batista CLC, Ribeiro VS, Nacimento MDSB. Influencia do uso de chupetas e mamadeiras na prática do aleitamento materno. J Health Biol Sci. 2017 Abr-Jun; 5(2): 184-191. 